\title{
20. Photoaktivierung von Cholesterin, Fetten und anderen Substanzen durch X-Strahlen.
}

\section{Von Sadayuki Havano.}

Biochemisches Laboratorium des Instituts für physikalische und Chemische Forschung, Tokyo.

(Rec. Nov. 12, 1925. Comm. by Umetaro SuzukI, M.I.A., Dec. 12, 1925.)

Der Verfasser hat nouerdings beobachtet, dass Cholesterin, Ölsäure, Lebertran, Balsam, Campher, Abietinsäure etc. nach der Bestrahlung mit Ultraviolettstrahlen im Dunkeln auf photographische Platte reagieren. (J. Agr. Chem. Soc. Japan, I (1925), No. 10.

Diese Wirkung tritt am stärksten im Gegenwart von Sauerstoff auf. Die gesättigten Fettsäuren, Traubenzucker, Rohrzucker, Stärke, Aminosäuren und Eiweisstoffe etc. zeigten aber keine Photowirkung nach der Bestrahlung.

Der Verfasser hat nun dieselben Versuche mit X-Strahlen angestellt and ähnliche Resultate erhalten.

Zu diesem Zweck wurden die X-Strahlen von einem Coolidge'schen Rohr entwickelt, welches mit Tungsten-Antikathode versehen war. Während des Betriebs war die Potentialdifferenz 30,000 Volts and die Stromstärke betrug $\overline{0}-6$ Milliamperes.

Ein Gramm der sorgiäitig gereinigten und getrockmeten Substanz von Cholesterin oder Borneol wurde in ein gewöhnliches Reagenzglas gefüllt, das offene Ende zugeschmolzen, mit dünnem Stanniolpapier vom $0,015 \mathrm{~mm}$ Dicke doppelt umwickelt und in der Entfernnung von $17 \mathrm{~cm}$ von der Strahlenquelle fünf Stunden lang andauernd mit $\mathrm{X}$-Strahlen bestrahlt. Hierauf wurde die Substanz herausgenmmen, in eine kleine Krystallisierschale gefüllt $(\mathrm{h}=1.6 \mathrm{~cm}, \mathrm{D}=2.7 \mathrm{~cm})$, mit einer Photoplatte (Lion Express Ortho) bedeckt und in einem vollkommen dunklen Raum 24 Stunden lang stehen gelassen. Nach dieser Zeit wurden das Negativ in gewöhnlicher' Weise entwickelt. Auf diese Weise wurde gefunden, dass Cholesterin, Borneol und Elaidinsäure stark auf Photoplatten reagierten, wïhrend die unbestralulten Substanzen keine 
No. 2.] Photoaktivierung von Chlosterin, Fetten und anderen Susbtanzen etc.

Wirkung darauf hervorbrachten. Auch Ölsäure und Lebertran, die ursprünglich nur schwache Wirkung hatten, reagierten nach der Bestrahlung bedeutend stärker. Wird das Reagenzrohr mit Kohlensäure gefüllt anstatt Luft oder Sauerstoff, so beobachtet man nach der Bestrahlung keine Photowirkung.

Ob die mit X-Strahlen aktivierten Substanzen antirachitische Wirkung auf Tiere entfalten, wie bei der Ultraviolett-bestrahlung, muss später untersucht werden.

Weitere Versuche sind im Gange. 